\title{
General fractional multiparameter white noise theory and stochastic partial differential equations
}

\author{
Yaozhong $\mathrm{Hu}^{1} \quad$ Bernt $\varnothing_{\mathrm{ksendal}}{ }^{2,3} \quad$ Tusheng Zhang ${ }^{4}$
}

\begin{abstract}
We present a white noise calculus for $d$-parameter fractional Brownian motion $B_{H}(x, \omega) ; x \in \mathbb{R}^{d}, \omega \in \Omega$ with general $d$-dimensional Hurst parameter $H=\left(H_{1}, \ldots, H_{d}\right)$ $\in(0,1)^{d}$. As an illustration we solve the stochastic Poisson problem $\Delta U(x)=-W_{H}(x)$; $x \in D, U=0$ on $\partial D$, where the potential $W_{H}(x)$ is $d$-parameter fractional white noise given by $W_{H}(x)=\frac{\partial^{d} B_{H}(x)}{\partial x_{1} \ldots \partial x_{d}}$, and $D \subset \mathbb{R}^{d}$ is a given bounded smooth domain. We also solve the linear stochastic heat equation $\frac{\partial U}{\partial t}(t, x)=\frac{1}{2} \Delta U(t, x)+W_{H}(t, x)$. For each equation we give sufficient conditions that the solutions $U(x)$ and $U(t, x)$, respectively, are square integrable random variables for all $t, x$.
\end{abstract}

\section{Introduction}

Recall that a 1-parameter fractional Brownian motion ( $\mathrm{fBm}$ ) with Hurst parameter $H \in$ $(0,1)$ is a Gaussian stochastic process $B_{H}(t)=B_{H}(t, \omega) ; t \in \mathbb{R}, \omega \in \Omega$ on a filtered probability space $(\Omega, \mathcal{F}, P)$ with the mean

$$
E\left[B_{H}(t)\right]=B_{H}(0)=0 \quad \text { for all } t \in \mathbb{R}
$$

and covariance

$$
E\left[B_{H}(s) B_{H}(t)\right]=\frac{1}{2}\left\{|s|^{2 H}+|t|^{2 H}-|s-t|^{2 H}\right\} \quad \text { for all } s, t \in \mathbb{R},
$$

where $E$ denotes expectation with respect to $P$. Note that if $H=\frac{1}{2}$ then $B_{H}(t)$ coincides with the classical Brownian motion.

For any $H \in(0,1)$ the process $B_{H}(t)$ is $H$-self-similar, in the sense that the law of $\left\{B_{H}(\alpha t)\right\}_{t \in \mathbb{R}}$ is the same as the law of $\left\{\alpha^{H} B_{H}(t)\right\}_{t \in \mathbb{R}}$ for all $\alpha>0$.

One of the reasons of the interest of fractional Brownian motion is that it can be used to model random phenomena with memory.

\footnotetext{
1 Dept. of Mathematics, University of Kansas, 405 Snow Hall, Lawrence, Kansas 66045-2142, USA, email: hu@math . ukans .edu

${ }^{2}$ Dept. of Mathematics, University of Oslo, P. O. Box 1053 Blindern, N-0316 Oslo, Norway, email:oksendal@math.uio.no

${ }^{3}$ Norwegian School of Economics and Business Administration, Helleveien 30, N-5045 Bergen, Norway

4 Dept. of Mathematics, University of Manchester, Oxford Road, Manchester M13 9PL, UK, email: tzhang@maths.man .ac.uk
} 
For example, if $\frac{1}{2}<H<1$ then $B_{H}(t)$ has a long range dependence, in the sense that

$$
\sum_{n=1}^{\infty} E\left[B_{H}(1)\left(B_{H}(n+1)-B_{H}(n)\right)\right]=\infty
$$

In this case the process is persistent, in the sense that high values have a tendency to be followed by an increase and low values by a decrease. This type of behavior is often observed in the levels of rivers, the characters of solar activity, the widths of consecutive annual rings and in the values of log returns in finance.

Similarly, if $0<H<\frac{1}{2}$ then

$$
E\left[B_{H}(1)\left(B_{H}(n+1)-B_{H}(n)\right)\right]<0
$$

and the process is anti-persistent, in the sense that high values have a tendency to be followed by a decrease and low values by an increase. This feature makes the process natural for turbulence modeling. Indeed, fractional Brownian motion was first introduced by Kolmogorov in 1940 (see [Ko]), in connection with turbulence studies. In 1968 the process was reintroduced by Mandelbrot and van Ness [MvN], who gave the process its current name and suggested a number of applications.

For more information on 1-parameter fractional Brownian motion we refer to the book by Shiryaev [S] and the references therein.

There is a natural generalization of $f B m$ to the multi-parameter case:

Fix a parameter dimension $d \in \mathbb{N}$ and a Hurst parameter $H=\left(H_{1}, H_{2}, \ldots, H_{d}\right) \in(0,1)^{d}$. Then we define the $d$-parameter fractional Brownian motion (or fractional Brownian field) $B_{H}\left(x_{1}, \ldots, x_{d}\right) ; x=\left(x_{1}, \ldots, x_{d}\right) \in \mathbb{R}^{d}$ as the Gaussian process (field) with mean

$$
E\left[B_{H}(x)\right]=B_{H}(0)=0 \quad \text { for all } x \in \mathbb{R}^{d}
$$

and covariance

$$
E\left[B_{H}(x) B_{H}(y)\right]=\left(\frac{1}{2}\right)^{d} \prod_{i=1}^{d}\left(\left|x_{i}\right|^{2 H_{i}}+\left|y_{i}\right|^{2 H_{i}}-\left|x_{i}-y_{i}\right|^{2 H_{i}}\right) \quad \text { for all } x, y \in \mathbb{R}^{d}
$$

These stochastic processes have been suggested in the modeling of the shape of mountain ranges $(d=2)$, the density of clouds $(d=3)$ and many other quantities. We refer to [AF] and $[\mathrm{M}]$ for more examples of modeling by multi-parameter $f \mathrm{Bm}$.

A stochastic calculus for 1-parameter $f B m$ based on the Wick-Itô integral was constructed by $[\mathrm{DHP}]$ in the case $\frac{1}{2}<H<1$. This was generalized to a fractional white noise calculus in $[\mathrm{H} \varnothing]$, still for the case $\frac{1}{2}<H<1$. Subsequently this 1-dimensional theory was extended (with certain restrictions) to be valid for all Hurst coefficients $H \in(0,1)$ by [EvdH].

A multi-parameter fractional white noise calculus was developed in [H1], [H2] and subsequently in [HØZ1] and [ØZ], where it was used to solve certain stochastic partial differential equations driven by multi-parameter fractional white noise $W_{H}(x)$. However, the presentation in all these papers was based on the assumption that $H=\left(H_{1}, \ldots, H_{d}\right) \in\left(\frac{1}{2}, 1\right)^{d}$.

The purpose of this paper is to present a multi-parameter fractional white noise theory valid for all Hurst parameters $H \in(0,1)^{d}$. This is achieved in Section 2 and Section 3 by making a synthesis of the 1-parameter approach of [EvdH] and the multi-parameter 
approach of [H1], [H2], [HØZ1] and [ØZ]. Then in Section 4 the theory is illustrated by solving explicitly the stochastic fractional Poisson equation

$$
\begin{aligned}
& \Delta U(x)=-W_{H}(x) ; \quad x \in D \subset \mathbb{R}^{d} \\
& U(x)=0 ; \quad x \in \partial D
\end{aligned}
$$

where $D$ is a given bounded domain in $\mathbb{R}^{d}$ with smooth boundary $\partial D$ and $W_{H}(x)=\frac{\partial^{d} B_{H}(x)}{\partial x_{1} \ldots \partial x_{d}}$ is $d$-parameter fractional white noise.

In section 5 we solve the stochastic fractional heat equation

$$
\begin{aligned}
& \frac{\partial U}{\partial t}(t, x)=\frac{1}{2} \Delta U(t, x)+W_{H}(t, x) ; \quad t \in(0, \infty), x \in D \subset \mathbb{R}^{d} \\
& U(0, x)=0 ; \quad x \in D \\
& U(t, x)=0 \quad t \geq 0, x \in \partial D .
\end{aligned}
$$

For both equations we find sufficient conditions that the solution is a square integrable random variable.

\section{Multiparameter fractional Brownian motion}

We start by recalling the standard white noise construction of multiparameter classical Brownian motion $B(x) ; x \in \mathbb{R}^{d}$. We refer to [HKPS], [HØUZ] and [Ku] for more details.

Let $\mathcal{S}=\mathcal{S}\left(\mathbb{R}^{d}\right)$ be the Schwartz space of rapidly decreasing smooth functions on $\mathbb{R}^{d}$ and let $\Omega:=\mathcal{S}^{\prime}\left(\mathbb{R}^{d}\right)$ be its dual, usually called the space of tempered distributions. By the Bochner-Minlos theorem there exists a probability measure $\mu$ on the Borel $\sigma$-algebra $\mathcal{B}(\Omega)$ such that

$$
\int_{\Omega} e^{i\langle\omega, f\rangle} d \mu(\omega)=e^{-\frac{1}{2}\|f\|^{2}} ; \quad f \in \mathcal{S}\left(\mathbb{R}^{d}\right)
$$

where $\langle\omega, f\rangle=\omega(f)$ denotes the action of $\omega \in \Omega=\mathcal{S}^{\prime}\left(\mathbb{R}^{d}\right)$ applied to $f \in \mathcal{S}\left(\mathbb{R}^{d}\right)$ and $\|f\|^{2}=\int_{\mathbb{R}^{d}}|f(x)|^{2} d x=\|f\|_{L^{2}\left(\mathbb{R}^{d}\right)}^{2}$. From (2.1) one can deduce that

$$
E_{\mu}[\langle\omega, f\rangle]=0 \quad \text { for all } f \in \mathcal{S}\left(\mathbb{R}^{d}\right)
$$

where $E_{\mu}$ denotes the expectation with respect to $\mu$. Moreover, we have the isometry

$$
E_{\mu}[\langle\omega, f\rangle\langle\omega, g\rangle]=(f, g)_{L^{2}\left(\mathbb{R}^{d}\right)} ; \quad f, g \in \mathcal{S}\left(\mathbb{R}^{d}\right)
$$

Using this isometry we can extend the definition of $\langle\omega, f\rangle \in L^{2}(\mu)$ from $\mathcal{S}\left(\mathbb{R}^{d}\right)$ to $L^{2}\left(\mathbb{R}^{d}\right)$ as follows:

$$
\left.\langle\omega, f\rangle=\lim _{n \rightarrow \infty}\left\langle\omega, f_{n}\right\rangle \quad \text { (limit in } L^{2}(\mu)\right)
$$

when $f_{n} \in \mathcal{S}\left(\mathbb{R}^{d}\right), f_{n} \rightarrow f \in L^{2}\left(\mathbb{R}^{d}\right)$ (limit in $L^{2}\left(\mathbb{R}^{d}\right)$ ).

In particular, we can now define, for $x=\left(x_{1}, \ldots, x_{d}\right) \in \mathbb{R}^{d}$,

$$
\widetilde{B}(x)=\widetilde{B}(x, \omega)=\left\langle\omega, \mathcal{X}_{[0, x]}(\cdot)\right\rangle ; \quad \omega \in \Omega
$$


where

$$
\mathcal{X}_{[0, x]}(y)=\prod_{i=1}^{d} \mathcal{X}_{\left[0, x_{i}\right]}\left(y_{i}\right) \quad \text { for } y=\left(y_{1}, \ldots, y_{d}\right) \in \mathbb{R}^{d}
$$

and

$$
\mathcal{X}_{\left[0, x_{i}\right]}\left(y_{i}\right)= \begin{cases}1 & \text { if } \quad 0 \leq y_{i} \leq x_{i} \\ -1 & \text { if } \quad x_{i} \leq y_{i} \leq 0, \text { except } x_{i}=y_{i}=0 \\ 0 & \text { otherwise }\end{cases}
$$

By Kolmogorov's continuity theorem the process $\{\widetilde{B}(x)\}$ has a continuous version which we will denote by $\{B(x)\}$. By $(2.1)-(2.3)$ it follows that $\{B(x)\}$ is a Gaussian process with mean

$$
E[B(x)]=B(0)=0
$$

and covariance (using (2.3))

$$
E[B(x) B(y)]=\left(\mathcal{X}_{[0, x]}, \mathcal{X}_{[0, y]}\right)_{L^{2}\left(\mathbb{R}^{d}\right)}= \begin{cases}\prod_{i=1}^{d} x_{i} \wedge y_{i} & \text { if } \quad x_{i}, y_{i} \geq 0 \text { for all } i \\ \prod_{i=1}^{d}\left(-x_{i}\right) \wedge\left(-y_{i}\right) & \text { if } \quad x_{i}, y_{i} \leq 0 \text { for all } i \\ 0 & \text { otherwise }\end{cases}
$$

Therefore $\{B(x)\}_{x \in \mathbb{R}^{d}}$ is a $d$-parameter Brownian motion.

We now use this Brownian motion to construct $d$-parameter fractional Brownian motion $B_{H}(x)$ for all Hurst parameters $H=\left(H_{1}, \ldots, H_{d}\right) \in(0,1)^{d}$. We do this by extending the procedure of $[\mathrm{EvdH}]$ to the $d$-dimensional case, as explained in [HØZ2]. For completeness we give the details.

For $0<H_{j}<1$ put

$$
K_{j}=k_{j}\left[2 \Gamma\left(H_{j}-\frac{1}{2}\right) \cos \left(\frac{\pi}{2}\left(H_{j}-\frac{1}{2}\right)\right)\right]^{-1}, \quad k_{j}=\sin \left(\pi H_{j}\right) \Gamma\left(2 H_{j}+1\right)
$$

and if $g \in \mathcal{S}\left(\mathbb{R}^{d}\right), x=\left(x_{1}, \ldots, x_{d}\right) \in \mathbb{R}^{d}$, define $m_{j} g(\cdot): \mathbb{R}^{d} \rightarrow \mathbb{R}$ by

$$
m_{j} g(x)=\left\{\begin{array}{lll}
K_{j} \int_{\mathbb{R}} \frac{g\left(x-t \varepsilon^{(j)}\right)-g(x)}{|t|^{\frac{3}{2}-H_{j}}} d t & \text { if } & 0<H_{j}<\frac{1}{2} \\
g(x) & \text { if } \quad H_{j}=\frac{1}{2} \\
K_{j} \int_{\mathbb{R}} \frac{g\left(x_{1}, \ldots, x_{j-1}, t, x_{j+1}, \ldots, x_{d}\right) d t}{\left|x_{j}-t\right|^{\frac{3}{2}-H_{j}}} & \text { if } \frac{1}{2}<H_{j}<1
\end{array}\right.
$$

where

$$
\varepsilon^{(j)}=(0,0, \ldots, 1, \ldots, 0), \quad \text { the } j \text { 'th unit vector. }
$$

Then define

$$
M_{H} f(x)=m_{1}\left(m_{2}\left(\ldots\left(m_{d-1}\left(m_{d} f\right)\right) \ldots\right)\right)(x) ; \quad f \in \mathcal{S}\left(\mathbb{R}^{d}\right)
$$


Note that if $f(x)=f_{1}(x) \ldots f_{d}\left(x_{d}\right)=:\left(f_{1} \otimes \cdots \otimes f_{d}\right)(x)$ is a tensor product, then

$$
M_{H} f(x)=\prod_{j=1}^{d}\left(M_{H_{j}} f_{j}\right)\left(x_{j}\right)
$$

where

$$
M_{H_{j}} f_{j}\left(x_{j}\right)=\left\{\begin{array}{lll}
K_{j} \int_{\mathbb{R}} \frac{f_{j}\left(x_{j}-t\right)-f_{j}\left(x_{j}\right)}{|t|^{\frac{3}{2}-H_{j}}} d t & ; \quad 0<H_{j}<\frac{1}{2} \\
f_{j}\left(x_{j}\right) & ; \quad H_{j}=\frac{1}{2} \\
K_{j} \int_{\mathbb{R}} \frac{f_{j}(t) d t}{\left|t-x_{j}\right|^{\frac{3}{2}-H_{j}}} & ; \quad \frac{1}{2}<H_{j}<1
\end{array}\right.
$$

Therefore, if

$$
\mathcal{F} g(\xi):=\hat{g}(\xi):=\int_{\mathbb{R}^{d}} e^{-i x \cdot \xi} g(x) d x ; \quad \xi=\left(\xi_{1}, \ldots, \xi_{d}\right) \in \mathbb{R}^{d}
$$

denotes the Fourier transform of $g$, we have by (2.13)

$$
\widehat{M_{H} f}(\xi)=\prod_{j=1}^{d} \widehat{M_{H_{j}} f_{j}}\left(\xi_{j}\right)=\prod_{j=1}^{d} k_{j}\left|\xi_{j}\right|^{\frac{1}{2}-H_{j}} \hat{f}_{j}\left(\xi_{j}\right)
$$

and

$$
\widehat{M_{H}^{-1} f}(\xi)=\left(\prod_{j=1}^{d} k_{j}\left|\xi_{j}\right|^{\frac{1}{2}-H_{j}}\right)^{-1} \hat{f}(\xi)
$$

For more information about the operator $M_{H}$ (for $d=1$ ) see [EvdH, Appendix].

We now construct $d$-parameter fractional Brownian motion $B_{H}(x)$ with Hurst parameter $H=\left(H_{1}, \ldots, H_{d}\right) \in(0,1)^{d}$ as follows:

First define

$$
\widetilde{B}_{H}(x)=\widetilde{B}_{H}(x, \omega)=\left\langle\omega, M_{H}\left(\mathcal{X}_{[0, x]}(\cdot)\right)\right\rangle
$$

with $\mathcal{X}_{[0, x]}(\cdot)$ as in $(2.5)-(2.6)$. Then $\widetilde{B}_{H}(x)$ is a Gaussian process with mean

$$
E\left[\widetilde{B}_{H}(x)\right]=\widetilde{B}_{H}(0)=0
$$

and covariance (using (2.13) and [EvdH, (1.13)])

$$
\begin{aligned}
& E\left[\widetilde{B}_{H}(x) \widetilde{B}_{H}(y)\right]=\int_{\mathbb{R}^{d}} M_{H}\left(\mathcal{X}_{[0, x]}(z)\right) M_{H}\left(\mathcal{X}_{[0, y]}(z)\right) d z \\
& \quad=\int_{\mathbb{R}^{d}} \prod_{i=1}^{d} M_{H_{i}} \mathcal{X}_{\left[0, x_{i}\right]}\left(z_{i}\right) \cdot \prod_{j=1}^{d} M_{H_{j}} \mathcal{X}_{\left[0, y_{j}\right]}\left(z_{j}\right) d z_{1} \ldots d z_{d} \\
& \quad=\prod_{j=1}^{d} \int_{\mathbb{R}} M_{H_{j}} \mathcal{X}_{\left[0, x_{j}\right]}(t) \cdot M_{H_{j}} \mathcal{X}_{\left[0, y_{j}\right]}(t) d t \\
& \quad=\left(\frac{1}{2}\right)^{d} \prod_{j=1}^{d}\left\{\left|x_{j}\right|^{2 H_{j}}+\left|y_{j}\right|^{2 H_{j}}-\left|x_{j}-y_{j}\right|^{2 H_{j}}\right\} ; \quad x, y \in \mathbb{R}^{d} .
\end{aligned}
$$


By Kolmogorov's continuity theorem we get that $\left\{\widetilde{B}_{H}(x)\right\}$ has a continuous version, which we denote by $\left\{B_{H}(x)\right\}$. From (2.18), (2.19) we conclude that $B_{H}(x)$ is a $d$-parameter fractional Brownian motion with Hurst parameter $H=\left(H_{1}, \ldots, H_{d}\right) \in(0,1)^{d}$.

If $f$ is a simple deterministic function of the form

$$
f(x)=\sum_{j=1}^{N} a_{j} \chi_{\left[0, y^{(j)}\right]}(x) ; \quad x \in \mathbb{R}^{d}
$$

for some $a_{j} \in \mathbb{R}, y^{(j)} \in \mathbb{R}^{d}$ and $N \in \mathbb{N}$, then we define its integral with respect to $B_{H}$ by

$$
\int_{\mathbb{R}^{d}} f(x) d B_{H}(x)=\sum_{j=1}^{N} a_{j} B_{H}\left(y^{(j)}\right) .
$$

Note that by (2.16) this coincides with $\left\langle\omega, M_{H} f>\right.$, and we have the isometry

$$
E\left[\left(\int_{\mathbb{R}^{d}} f(x) d B_{H}(x)\right)^{2}\right]=E\left[<\omega, M_{H} f>^{2}\right]=\left\|M_{H} f\right\|_{L^{2}\left(\mathbb{R}^{d}\right)}^{2} .
$$

We can extend the definition of this integral to all $g \in L_{H}^{2}\left(\mathbb{R}^{d}\right)$, where

$$
L_{H}^{2}\left(\mathbb{R}^{d}\right)=\left\{g: \mathbb{R}^{d} \rightarrow \mathbb{R} ;\|g\|_{L_{H}^{2}\left(\mathbb{R}^{d}\right)}:=\left\|M_{H} g\right\|_{L^{2}\left(\mathbb{R}^{d}\right)}<\infty\right\} .
$$

by setting

$$
\int_{\mathbb{R}^{d}} g(x) d B_{H}(x):=\left\langle\omega, M_{H} g\right\rangle \quad \text { for all } g \in L_{H}^{2}\left(\mathbb{R}^{d}\right)
$$

Moreover, if $f, g \in L_{H}^{2}\left(\mathbb{R}^{d}\right)$ then we have the isometry

$$
\begin{gathered}
E\left[\left(\int_{\mathbb{R}^{d}} f(x) d B_{H}(x)\right)\left(\int_{\mathbb{R}^{d}} g(x) d B_{H}(x)\right)\right]=E\left[\left\langle\omega, M_{H} f\right\rangle\left\langle\omega, M_{H} g\right\rangle\right] \\
=\left(M_{H} f, M_{H} g\right)_{L^{2}\left(\mathbb{R}^{d}\right)}=(f, g)_{L_{H}^{2}\left(\mathbb{R}^{d}\right)} .
\end{gathered}
$$

We mention that the space $L_{H}^{2}\left(\mathbb{R}^{d}\right)$ is not complete for $H>\frac{1}{2}$, see [H3], [PT] for more details.

\section{Multiparameter fractional white noise calculus}

With the processes $B_{H}(x)$ constructed in Section 2 as a starting point we proceed to develop a $d$-parameter white noise theory as in [HØZ1] and [ØZ], but modified according to the 1-parameter approach in $[\mathrm{EvdH}]$.

Let

$$
h_{n}(t)=(-1)^{n} e^{\frac{t^{2}}{2}} \frac{d^{n}}{d t^{n}}\left(e^{-\frac{t^{2}}{2}}\right) ; \quad n=0,1,2, \ldots ; t \in \mathbb{R}
$$

be the Hermite polynomials and let

$$
\tilde{h}_{n}(t)=\pi^{-\frac{1}{4}}((n-1) !)^{-\frac{1}{2}} h_{n-1}(\sqrt{2} t) e^{-\frac{t^{2}}{2}} ; \quad n=1,2, \ldots ; t \in \mathbb{R}
$$


be the Hermite functions.

If $\alpha=\left(\alpha_{1}, \ldots, \alpha_{d}\right) \in \mathbb{N}^{d}$ (with $\left.\mathbb{N}=\{1,2, \ldots\}\right)$ and $x=\left(x_{1}, \ldots, x_{d}\right) \in \mathbb{R}^{d}$ define

$$
\eta_{\alpha}(x)=\tilde{h}_{\alpha_{1}}\left(x_{1}\right) \ldots \tilde{h}_{\alpha_{d}}\left(x_{d}\right)=\left(\tilde{h}_{\alpha_{1}} \otimes \cdots \otimes \tilde{h}_{\alpha_{d}}\right)(x)
$$

and

$$
e_{\alpha}(x)=\left(M_{H_{\alpha_{1}}}^{-1} \tilde{h}_{\alpha_{1}}\right)\left(x_{1}\right) \ldots\left(M_{H_{\alpha_{d}}}^{-1} \tilde{h}_{\alpha_{d}}\right)\left(x_{d}\right)=\left(M_{H}^{-1} \eta_{\alpha}\right)(x)
$$

Let $\left\{\alpha^{(i)}\right\}_{i=1}^{\infty}$ be a fixed ordering of $\mathbb{N}^{d}$ with the property that, with $\left|\alpha^{(i)}\right|=\alpha_{1}^{(i)}+\cdots+\alpha_{d}^{(i)}$,

$$
i<j \Rightarrow\left|\alpha^{(i)}\right| \leq\left|\alpha^{(j)}\right|
$$

Note that this implies that there exists a constant $C<\infty$ such that

$$
\left|\alpha^{(k)}\right| \leq C k \quad \text { for all } k \text {. }
$$

With a slight abuse of notation let us write

$$
\eta_{n}(x):=\eta_{\alpha^{(n)}}(x)=M_{H} e_{n}(x)
$$

and

$$
e_{n}(x):=e_{\alpha^{(n)}}(x)=M_{H}^{-1} \eta_{n}(x) ; \quad n=1,2, \ldots
$$

Now let $\mathcal{J}=\left(\mathbb{N}_{0}^{\mathbb{N}}\right)_{c}$ denote the set of all finite sequences $\alpha=\left(\alpha_{1}, \ldots, \alpha_{m}\right)$ with $\alpha_{j} \in \mathbb{N}_{0}=$ $\mathbb{N} \cup\{0\}, m=1,2, \ldots$ Then if $\alpha=\left(\alpha_{1}, \ldots, \alpha_{m}\right) \in \mathcal{J}$ we define

$$
\mathcal{H}_{\alpha}(\omega)=h_{\alpha_{1}}\left(\left\langle\omega, \eta_{1}\right\rangle\right) \ldots h_{\alpha_{m}}\left(\left\langle\omega, \eta_{m}\right\rangle\right)
$$

In particular, note that by (2.21) we have

$$
\begin{aligned}
\mathcal{H}_{\varepsilon^{(i)}}(\omega) & =h_{1}\left(\left\langle\omega, \eta_{i}\right\rangle\right)=\left\langle\omega, \eta_{i}\right\rangle=\int_{\mathbb{R}^{d}} \eta_{i}(x) d B(x) \\
& =\int_{\mathbb{R}^{d}} M_{H} e_{i}(x) d B(x)=\left\langle\omega, M_{H} e_{i}\right\rangle=\int_{\mathbb{R}^{d}} e_{i}(x) d B_{H}(x) ; \quad i=1,2, \ldots
\end{aligned}
$$

We recall the following well-known result:

\section{Theorem 3.1 (The chaos expansion theorem)}

Every $F \in L^{2}(\mu)$ can be written as the form

$$
F(\omega)=\sum_{\alpha \in \mathcal{J}} c_{\alpha} \mathcal{H}_{\alpha}(\omega)
$$

where $c_{\alpha} \in \mathbb{R}$. Moreover, we have the isometry

$$
\|F\|_{L^{2}(\mu)}^{2}=\sum_{\alpha \in \mathcal{J}} \alpha ! c_{\alpha}^{2}
$$

where $\alpha !=\alpha_{1} ! \alpha_{2} ! \ldots \alpha_{m} !$ if $\alpha=\left(\alpha_{1}, \ldots, \alpha_{m}\right)$. 
Note that if $f \in \mathcal{S}\left(\mathbb{R}^{d}\right)$ then $M_{H} f \in L^{2}\left(\mathbb{R}^{d}\right)$. Moreover, if $f, g \in \mathcal{S}\left(\mathbb{R}^{d}\right)$ then

$$
\left(g, M_{H} f\right)_{L^{2}\left(\mathbb{R}^{d}\right)}=\left(\hat{g}, \widehat{M_{H} f}\right)_{L^{2}\left(\mathbb{R}^{d}\right)}=\left(M_{H} g, f\right)_{L^{2}\left(\mathbb{R}^{d}\right)}
$$

Therefore, since the action of $\omega \in \Omega=\mathcal{S}^{\prime}\left(R^{d}\right)$ extends to $L^{2}\left(R^{d}\right)$, we can extend the definition of the operator $M_{H}$ from $\mathcal{S}\left(\mathbb{R}^{d}\right)$ to $\Omega=\mathcal{S}^{\prime}\left(\mathbb{R}^{d}\right)$ by setting

$$
\left\langle M_{H} \omega, f\right\rangle=\left\langle\omega, M_{H} f\right\rangle ; \quad f \in \mathcal{S}(\mathbb{R}), \omega \in \mathcal{S}^{\prime}(\mathbb{R})
$$

We now define

$$
L_{H}^{2}(\mu)=\left\{G: \Omega \rightarrow \mathbb{R} ; G \circ M_{H} \in L^{2}(\mu)\right\}
$$

and

$$
\|G\|_{L_{H}^{2}(\mu)}^{2}=\left\|G \circ M_{H}\right\|_{L^{2}(\mu)}^{2} \quad \text { for } G \in L_{H}^{2}(\mu) .
$$

Example 3.2 The chaos expansion of classical Brownian motion $B(x) \in L^{2}(\mu)$ is

$$
B(x)=\left\langle\omega, \mathcal{X}_{[0, x]}\right\rangle=\sum_{k=1}^{\infty}\left(\mathcal{X}_{[0, x]}, \eta_{k}\right)_{L^{2}\left(\mathbb{R}^{d}\right)}\left\langle\omega, \eta_{k}\right\rangle=\sum_{k=1}^{\infty}\left(\int_{-\infty}^{x} \eta_{k}(y) d y\right) \cdot \mathcal{H}_{\varepsilon^{(k)}}(\omega)
$$

where in general we put

$$
\int_{-\infty}^{x} g(y) d y=\int_{-\infty}^{x_{d}} \cdots \int_{-\infty}^{x_{1}} g(y) d y_{1} \ldots d y_{d} ; \quad x=\left(x_{1}, \ldots, x_{d}\right) \in \mathbb{R}^{d} .
$$

Hence by $(2.17)$ the chaos expansion of fractional Brownian motion $B_{H}(x) \in L_{H}^{2}(\mu)$ is

$$
\begin{aligned}
B_{H}(x) & =\left\langle\omega, M_{H} \mathcal{X}_{[0, x]}\right\rangle \\
& =\sum_{k=1}^{\infty}\left(M_{H} \mathcal{X}_{[0, x]}, \eta_{k}\right)_{L^{2}\left(\mathbb{R}^{d}\right)}\left\langle\omega, \eta_{k}\right\rangle=\sum_{k=1}^{\infty}\left(\mathcal{X}_{[0, x]}, M_{H} \eta_{k}\right)_{L^{2}\left(\mathbb{R}^{d}\right)} \mathcal{H}_{\varepsilon^{(k)}}(\omega) \\
& =\sum_{k=1}^{\infty}\left(\int_{-\infty}^{x} M_{H} \eta_{k}(y) d y\right) \mathcal{H}_{\varepsilon(k)}(\omega)
\end{aligned}
$$

Similarly, if $f \in L_{H}^{2}\left(\mathbb{R}^{d}\right)$ then by $(2.21)$

$$
\int_{\mathbb{R}} f(x) d B_{H}(x)=\left\langle\omega, M_{H} f\right\rangle=\left\langle M_{H} \omega, f\right\rangle=\sum_{k=1}^{\infty}\left(M_{H} \eta_{k}, f\right)_{L^{2}\left(\mathbb{R}^{d}\right)} \mathcal{H}_{\varepsilon(k)}(\omega) .
$$

Next we define the $d$-parameter Hida test function and distribution spaces $(\mathcal{S})$ and $(\mathcal{S})^{*}$, respectively:

\section{Definition 3.3}

a) For $k=1,2, \ldots$ let $(\mathcal{S})^{(k)}$ be the set of $G \in L^{2}(\mu)$ with expansion

$$
G(\omega)=\sum_{\alpha} c_{\alpha} \mathcal{H}_{\alpha}(\omega)
$$


such that

$$
\|G\|_{(\mathcal{S})^{(k)}}^{2}:=\sum_{\alpha} \alpha ! c_{\alpha}^{2}(2 \mathbb{N})^{\alpha k}<\infty
$$

where

$$
(2 \mathbb{N})^{\beta}=(2 \cdot 1)^{\beta_{1}}(2 \cdot 2)^{\beta_{2}} \cdots(2 m)^{\beta_{m}} \quad \text { if } \quad \beta=\left(\beta_{1}, \ldots, \beta_{m}\right) \in \mathcal{J}
$$

The space of Hida test functions, $(\mathcal{S})$, is defined by

$$
(\mathcal{S})=\bigcap_{k=1}^{\infty}(\mathcal{S})^{(k)}, \quad \text { equipped with the projective topology. }
$$

b) For $q=1,2, \ldots$ let $(\mathcal{S})^{(-q)}$ be the set of all formal expansions

$$
G=\sum_{\alpha} c_{\alpha} \mathcal{H}_{\alpha}(\omega)
$$

such that

$$
\|G\|_{(\mathcal{S})^{(-q)}}:=\sum_{\alpha} \alpha ! c_{\alpha}^{2}(2 \mathbb{N})^{-q \alpha}<\infty
$$

The space of Hida distributions, $(\mathcal{S})^{*}$, is defined by

$$
(\mathcal{S})^{*}=\bigcup_{q=1}^{\infty}(\mathcal{S})^{(-q)}, \quad \text { equipped with the inductive topology. }
$$

Note that with this definition we have

$$
(\mathcal{S}) \subset L^{2}(\mu) \subset(\mathcal{S})^{*}
$$

Example 3.4 Define fractional white noise, $W_{H}(x)$, by

$$
W_{H}(x)=\sum_{k=1}^{\infty} M_{H} \eta_{k}(x) \mathcal{H}_{\varepsilon(k)}(\omega) ; \quad x \in \mathbb{R}^{d} .
$$

Then $W_{H}(x) \in(\mathcal{S})^{*}$ because in this case, by (3.3) and (3.5),

$$
\begin{aligned}
\sum_{\alpha} \alpha ! c_{\alpha}^{2}(2 \mathbb{N})^{-q \alpha} & =\sum_{k=1}^{\infty}\left(M_{H} \eta_{k}\right)^{2}(x)(2 \mathbb{N})^{-q \varepsilon^{(k)}} \\
& =\sum_{k=1}^{\infty}\left(M_{H_{\alpha_{1}(k)}} \tilde{h}_{\alpha_{1}^{(k)}}\right)^{2}\left(x_{1}\right) \ldots\left(M_{H_{\alpha_{d}(k)}} \tilde{h}_{\alpha_{d}^{(k)}}\right)^{2}\left(x_{d}\right)(2 k)^{-q} \\
& \leq \sum_{k=1}^{\infty} C_{1}\left(\prod_{j=1}^{d}\left(\alpha_{j}^{(k)}\right)^{\frac{2}{3}-\frac{{ }^{\alpha_{j}^{(k)}}}{2}}\right)^{2}(2 k)^{-q} \leq C_{1} \sum_{k=1}^{\infty}(2 k)^{\frac{4 d}{3}-q}<\infty
\end{aligned}
$$

for $q>\frac{4 d}{3}+1\left(C_{1}\right.$ is a constant). Here we have used the estimate

$$
\left|M_{H_{j}} \tilde{h}_{n}(t)\right| \leq C_{2} n^{\frac{2}{3}-\frac{H_{j}}{2}} \quad \text { for all } t\left(C_{2} \text { constant }\right)
$$


from Section 3 of $[\mathrm{EvdH}]$.

Note that from (3.27) and (3.19) we have that

$$
\frac{\partial^{d}}{\partial x_{1} \ldots \partial x_{d}} B_{H}(x)=W_{H}(x) \quad\left(\text { in }(\mathcal{S})^{*}\right) \text { for all } x \in \mathbb{R}^{d} .
$$

This justifies the name fractional white noise for the process $W_{H}(x)$.

Choose $g \in \mathcal{S}\left(\mathbb{R}^{d}\right)$ and let $m_{j} g$ be as in (2.10). We establish a useful formula for the $L^{2}(\mathbb{R})$ norm of $m_{j} g$.

Theorem 3.5 Let $f$ and $g$ be elements in $\mathcal{S}(\mathbb{R})$ (the space of rapidly decreasing functions). If $0<H_{j}<\frac{1}{2}$, then there is a constant $\kappa$ such that

$$
\int_{\mathbb{R}} m_{j} f(x) m_{j} g(x) d x=\kappa \int_{\mathbb{R}} \int_{\mathbb{R}}|x-y|^{2 H_{j}} f^{\prime}(x) g^{\prime}(y) d x d y
$$

Proof. From (2.15) we have

$$
\mathcal{F}\left(m_{j} f\right)(\xi)=K_{j}|\xi|^{\frac{1}{2}-H_{j}} \hat{f}(\xi)
$$

Thus

$$
\int_{\mathbb{R}} m_{j} f(x) m_{j} g(x) d x=\frac{1}{2 \pi} K_{j}^{2} \int_{\mathbb{R}}|\xi|^{1-2 H_{j}} \overline{\hat{f}}(\xi) \hat{g}(\xi) d \xi
$$

For $\alpha>0$ define

$$
I^{\alpha} \phi(x)=\gamma_{\alpha} \int_{\mathbb{R}} \frac{\phi(t)}{|t-x|^{1-\alpha}} d t,
$$

where $\gamma_{\alpha}=2 \Gamma(\alpha) \cos (\alpha \pi / 2)$. By [SKM], we have

$$
\mathcal{F}\left(I^{\alpha} \phi\right)(\xi)=|\xi|^{-\alpha} \hat{\phi}(\xi)
$$

Therefore,

$$
\int_{\mathbb{R}} f^{\prime}(x) I^{\alpha} g^{\prime}(x) d x=\frac{1}{2 \pi} \int_{\mathbb{R}}|\xi|^{2-\alpha} \overline{\hat{f}}(\xi) \hat{g}(\xi) d \xi
$$

Hence,

$$
\int_{\mathbb{R}} m_{j} f(x) m_{j} g(x) d x=K_{j}^{2} \int_{\mathbb{R}} f^{\prime}(x) I^{\alpha} g^{\prime}(x) d x
$$

if $1-2 H_{j}=2-\alpha$. That is

$$
\alpha=1+2 H_{j} .
$$

When the above identity is true, we have

$$
\int_{\mathbb{R}} f^{\prime}(x) I^{\alpha} g^{\prime}(x) d x=\kappa \int_{\mathbb{R}^{2}}|x-y|^{2 H_{j}} f^{\prime}(x) g^{\prime}(y) d x d y,
$$

where

$$
\kappa=\gamma_{\alpha} K_{j}^{2}
$$

Remark It is easy to extend the identity to more general functions. 


\section{The stochastic fractional Poisson equation}

We now illustrate the use of the theory above by solving the Poisson equation with fractional white noise heat source:

Let $D \subset \mathbb{R}^{d}$ be a given bounded domain with smooth $\left(C^{\infty}\right)$ boundary. We want to find $U(\cdot): \bar{D} \rightarrow(\mathcal{S})^{*}$ such that

$$
\begin{aligned}
\Delta U(x) & =-W_{H}(x) & & \text { for } x \in D \\
U(x) & =0 & & \text { for } x \in \partial D
\end{aligned}
$$

(where $\Delta=\frac{1}{2} \sum_{i=1}^{d} \frac{\partial^{2}}{\partial x_{i}^{2}}$ is the Laplacian operator) and such that $U$ is continuous on the closure $\bar{D}$ of $D$.

From classical potential theory we are led to the solution candidate

$$
U(x)=\int_{D} G(x, y) W_{H}(y) d y=\int_{D} G(x, y) d B_{H}(y)
$$

where $G$ is the classical Green function for the Dirichlet Laplacian.

We first verify that $U(x) \in(\mathcal{S})^{*}$ for all $x$. To this end, consider the expansion of $U(x)$ :

$$
\begin{aligned}
U(x) & =\int_{D} G(x, y) \sum_{k=1}^{\infty} M_{H} \eta_{k}(y) \mathcal{H}_{\varepsilon^{(k)}}(\omega) d y \\
& =\sum_{k=1}^{\infty} a_{k}(x) \mathcal{H}_{\varepsilon^{(k)}}(\omega), \quad \text { where } \\
a_{k}(x) & =\int_{D} G(x, y) M_{H} \eta_{k}(y) d y .
\end{aligned}
$$

By the estimate (3.28) we have

$$
\left|a_{k}(x)\right| \leq C_{3} k^{\frac{2 d}{3}} \int_{D} G(x, y) d y \leq C_{4} k^{\frac{2 d}{3}},
$$

and therefore

$$
\sum_{k=1}^{\infty} a_{k}^{2}(x)(2 \mathbb{N})^{-q \varepsilon_{k}} \leq C_{4}^{2} \sum_{k=1}^{\infty}(2 k)^{\frac{4 d}{3}}(2 k)^{-q}<\infty
$$

for $q>\frac{4 d}{3}+1$.

This proves that $U(x) \in(\mathcal{S})^{*}$ and the same estimate gives that $U: \bar{D} \rightarrow(\mathcal{S})^{*}$ is continuous.

The proof that $\Delta U(x)=-W_{H}(x)$ is identical to the proof given in [HØZ1, Section 3] and is omitted. We conclude that $U(x)$ given by (4.3) is indeed the solution of (4.1)-(4.2).

Thus we have:

Theorem 4.1 Let $H=\left(H_{1}, \ldots, H_{d}\right) \in(0,1)^{d}$. The stochastic fractional Poisson equation (4.1)-(4.2) has a unique solution $U(x) \in(\mathcal{S})^{*}$ given by

$$
U(x)=\int_{D} G(x, y) d B_{H}(y)
$$

where $G(x, y)$ is the classical Green function for the Laplacian. 
Next we discuss when this solution $U(x)$ belongs to $L^{2}(\mu)$. In [HØZ1] it is proved that if

$$
\frac{1}{2}<H_{i}<1 \quad \text { for all } i
$$

and

$$
\sum_{i=1}^{d} H_{i}>d-2
$$

then $U(x) \in L^{2}(\mu)$ for all $x$.

We will show that condition (4.8) is sufficient to have $U(x) \in L^{2}(\mu)$ also without condition $(4.7)$ :

Theorem 4.2 Let $H=\left(H_{1}, \ldots, H_{d}\right) \in(0,1)^{d}$ and $\mathbb{H}:=\left\{i, H_{i}<1 / 2\right\}$. Suppose that $\sharp \mathbb{H} \leq 1$ and

$$
\sum_{i \in \mathbb{H}} H_{i}>d-2
$$

Then the solution $U(x)$ given by (4.6) belongs to $L^{2}(\mu)$ for all $x$.

Proof. By (4.3) and (2.22) we have

$$
\begin{aligned}
E\left[U^{2}(x)\right] & =(G(x, \cdot), G(x, \cdot))_{L_{H}^{2}\left(\mathbb{R}^{d}\right)} \\
& =\left(M_{H} G(x, \cdot), M_{H} G(x, \cdot)\right)_{L^{2}\left(\mathbb{R}^{d}\right)}=\int_{\mathbb{R}^{d}}\left(M_{H} G(x, y)\right)^{2} d y,
\end{aligned}
$$

where the operator $M_{H}$ acts on $y$, and we have extended the function $G(x, \cdot)$ and $M_{H} G(x, \cdot)$ to $\mathbb{R}^{d}$ by defining it to be zero outside $D$

Without loss of generality we can assume that

$$
H_{1}<\frac{1}{2} \quad \text { and } \quad H_{i}>\frac{1}{2} \quad \text { for } i>1 .
$$

Since $\partial D$ is smooth there exists a constant $C$ such that

$$
\left|\frac{\partial^{k}}{\partial y_{1} \ldots \partial y_{k}} G(x, y)\right| \leq C \frac{\prod_{i=1}^{k}\left|x_{i}-y_{i}\right|}{|x-y|^{d+2 k-2}} .
$$

Recall that if $\frac{1}{2}<H_{j}<1$,

$$
\int_{R} m_{j} f(x)^{2} d x=c_{j} \int_{R} \int_{R} f(x)|x-y|^{2 H_{j}-2} f(y) d x d y
$$

Hence by (4.10) and Theorem 3.8

$$
E\left[U^{2}(x)\right] \leq C \int_{D} \int_{D} \frac{\left|x_{1}-y_{1}\right|}{|x-y|^{d}} \phi(y, z) \frac{\left|x_{1}-z_{1}\right|}{|x-z|^{d}} d y d z
$$

where

$$
\phi(y, z)=\left|y_{1}-z_{1}\right|^{2 H_{1}} \prod_{i=2}^{d}\left|y_{i}-z_{i}\right|^{2 H_{i}-2}
$$


Let $z^{\prime}=x-z$ and $y^{\prime}=x-y$. Since $D$ is bounded there exists a positive constant $R$ such that, using (4.13),

$$
\begin{aligned}
E\left[U^{2}(x)\right] & \leq C \int_{-R}^{R} \cdots \int_{-R}^{R} \frac{\left|y_{1}^{\prime}\right|}{\left|y^{\prime}\right|} \phi\left(y^{\prime}, z^{\prime}\right) \frac{\left|z_{1}^{\prime}\right|}{\left|z^{\prime}\right|^{d}} d y^{\prime} d z^{\prime} \\
& =C \int_{-R}^{R} \cdots \int_{-R}^{R} \frac{\left|y_{1}\right|\left|z_{1}\right|\left|y_{1}-z_{1}\right|^{2 H_{1}}}{|y|^{d}} \frac{\prod_{i=2}^{d}\left|y_{i}-z_{i}\right|^{2 H_{i}-2}}{|z|^{d}} d y d z
\end{aligned}
$$

Next we are going to show that the integral above is finite.

For notational simplicity, we assume that $R=1$. For any $a>0, b>0, d>0$, we claim that there is a constant $c$, independent of $a$ and $b$ such that

$$
\int_{-1}^{1} \int_{-1}^{1} \frac{|y||z||y-z|^{2 H_{1}}}{\left(y^{2}+a\right)^{d / 2}\left(z^{2}+b\right)^{d / 2}} d y d z \leq c \frac{a^{H_{1}}+b^{H_{1}}}{a^{d / 2-1} b^{d / 2-1}}
$$

Indeed,

$$
\begin{aligned}
& \int_{-1}^{1} \int_{-1}^{1} \frac{|y||z||y-z|^{2 H_{1}}}{\left(y^{2}+a\right)^{d / 2}\left(z^{2}+b\right)^{d / 2}} d y d z \\
\leq & c\left\{\int_{-1}^{1} d y \int_{-1}^{1} \frac{|y||z|\left(|y|^{2 H_{1}}+|z|^{2 H_{1}}\right)}{\left(y^{2}+a\right)^{d / 2}\left(z^{2}+b\right)^{d / 2}} d z\right\} \\
\leq & c\left\{\int_{0}^{1} d y \frac{y^{2 H_{1}+1}}{\left(y^{2}+a\right)^{d / 2}} \int_{0}^{1} \frac{z}{\left(z^{2}+b\right)^{d / 2}} d z+\int_{0}^{1} d z \frac{z^{2 H_{1}+1}}{\left(z^{2}+b\right)^{d / 2}} \int_{0}^{1} \frac{y}{\left(y^{2}+a\right)^{d / 2}} d y\right\} \\
\leq & c \int_{0}^{1} \frac{y^{2 H_{1}+1}}{\left(y^{2}+a\right)^{d / 2}}\left[b^{1-d / 2}-(1+b)^{1-d / 2}\right] d y+c \int_{0}^{1} \frac{z^{2 H_{1}+1}}{\left(z^{2}+b\right)^{d / 2}}\left[a^{1-d / 2}-(1+a)^{1-d / 2}\right] d z \\
\leq & c b^{1-d / 2} \int_{0}^{1} \frac{z}{\left(y^{2}+a\right)^{d / 2-H_{1}}} d y+c a^{1-d / 2} \int_{0}^{1} \frac{a^{2}}{\left(z^{2}+b\right)^{d / 2-H_{1}}} d z \\
\leq & c b^{1-d / 2} a^{1-d / 2+H_{1}}+a^{1-d / 2} b^{1-d / 2+H_{1}}=c \frac{a^{H_{1}}+b^{H_{1}}}{a^{d / 2-1} b^{d / 2-1}} .
\end{aligned}
$$

Applying (4.16) for $a=\sum_{i=2}^{d} y_{i}^{2}, b=\sum_{i=2}^{d} z_{i}^{2}$, we have

$$
\begin{gathered}
\int_{-1}^{1} \cdots \int_{-1}^{1} \frac{\left|y_{1}\right|\left|z_{1}\right|\left|y_{1}-z_{1}\right|^{2 H_{1}}}{|y|^{d}} \frac{\prod_{i=2}^{d}\left|y_{i}-z_{i}\right|^{2 H_{i}-2}}{|z|^{d}} d y d z \\
=\int_{-1}^{1} \cdots \int_{-1}^{1} \prod_{i=2}^{d}\left|y_{i}-z_{i}\right|^{2 H_{i}-2} d y_{2} \cdots d y_{d} d z_{2} \cdots d z_{d} \int_{-1}^{1} \int_{-1}^{1} \frac{\left|y_{1}\right|\left|z_{1}\right|\left|y_{1}-z_{1}\right|^{2 H_{1}}}{|y|^{d}|z|^{d}} d y_{1} d z_{1} \\
\leq c \int_{-1}^{1} \cdots \int_{-1}^{1} \frac{\prod_{i=2}^{d}\left|y_{i}-z_{i}\right|^{2 H_{i}-2}\left[\left(\sum_{i=2}^{d} y_{i}^{2}\right)^{H_{1}}+\left(\sum_{i=2}^{d} z_{i}^{2}\right)^{H_{1}}\right]}{\left(\sum_{i=2}^{d} y_{i}^{2}\right)^{d / 2-1}\left(\sum_{i=2}^{d} z_{i}^{2}\right)^{d / 2-1}} d y_{2} \cdots d y_{d} d z_{2} \cdots d z_{d} \\
=\int_{-1}^{1} \cdots \int_{-1}^{1} \frac{\prod_{i=2}^{d}\left|y_{i}-z_{i}\right|^{2 H_{i}-2}}{\left(\sum_{i=2}^{d} y_{i}^{2}\right)^{d / 2-1-H_{1}}\left(\sum_{i=2}^{d} z_{i}^{2}\right)^{d / 2-1}} d y_{2} \cdots d y_{d} d z_{2} \cdots d z_{d}
\end{gathered}
$$




$$
\begin{gathered}
+\int_{-1}^{1} \cdots \int_{-1}^{1} \frac{\prod_{i=2}^{d}\left|y_{i}-z_{i}\right|^{2 H_{i}-2}}{\left(\sum_{i=2}^{d} y_{i}^{2}\right)^{d / 2-1}\left(\sum_{i=2}^{d} z_{i}^{2}\right)^{d / 2-1-H_{1}}} d y_{2} \cdots d y_{d} d z_{2} \cdots d z_{d} \\
=: I+I I .
\end{gathered}
$$

We now prove both $I$ and $I I$ are finite. By symmetry, we only look at $I$.

For any choice of positive numbers $\alpha_{i}>0$ with $\sum_{i=2}^{d} \alpha_{i}=1$ and positive numbers $\beta_{i}>0$ with $\sum_{i=2}^{d} \beta_{i}=1$, we have

$$
I \leq \int_{-1}^{1} \ldots \int_{-1}^{1} \frac{\prod_{i=2}^{d}\left|y_{i}-z_{i}\right|^{2 H_{i}-2}}{\prod_{i=2}^{d}\left|y_{i}\right|^{\alpha_{i}\left(d-2-2 H_{1}\right)}\left|z_{i}\right|^{\beta_{i}(d-2)}} d y_{2} \ldots d y_{d} d z_{2} \ldots d z_{d}
$$

So, $I$ is finite if the following conditions are met:

$$
\begin{aligned}
& \alpha_{i}\left(d-2-2 H_{1}\right)<1, \quad i=2, \cdots, d \\
& \beta_{i}(d-2)<1, \\
& 2 H_{i}+2<2, \quad i=2, \cdots, d \\
& \alpha_{i}\left(d-2-2 H_{1}\right)+\beta_{i}(d-2)-2, \cdots, d
\end{aligned}
$$

Adding these inequalities in (4.18), we see that it is sufficient to have

$$
2(d-2)-2 H_{1}<\sum_{i=2}^{d} 2 H_{i}
$$

namely,

$$
\sum_{i=1}^{d} H_{i}>d-2
$$

This completes the proof.

Remark It is natural to ask if condition (4.9) is also necessary to have $U(x) \in L^{2}(\mu)$. Now we give a discussion.

We need the following: For any $a>0, b>0, d>0$ ( $a$ and $b$ bounded), there is a constant $c$, independent of $a$ and $b$ such that

$$
\int_{-1}^{1} \int_{-1}^{1} \frac{|y||z||y-z|^{2 H_{1}}}{\left(y^{2}+a\right)^{d / 2}\left(z^{2}+b\right)^{d / 2}} d y d z \geq c \frac{a^{H_{1}}}{a^{d / 2-1} b^{d / 2-1}}
$$

and

$$
\int_{-1}^{1} \int_{-1}^{1} \frac{|y||z||y-z|^{2 H_{1}}}{\left(y^{2}+a\right)^{d / 2}\left(z^{2}+b\right)^{d / 2}} d y d z \geq c \frac{b^{H_{1}}}{a^{d / 2-1} b^{d / 2-1}}
$$


In fact,

$$
\begin{aligned}
& \int_{-1}^{1} \int_{-1}^{1} \frac{|y||z||y-z|^{2 H_{1}}}{\left(y^{2}+a\right)^{d / 2}\left(z^{2}+b\right)^{d / 2}} d y d z \\
\geq & \int_{0}^{1} \int_{-1}^{1} \frac{y|z||y-z|^{2 H_{1}}}{\left(y^{2}+a\right)^{d / 2}\left(z^{2}+b\right)^{d / 2}} d y d z \\
\geq & \int_{0}^{1} \int_{-1}^{0} \frac{y|z|^{2 H_{1}+1}}{\left(y^{2}+a\right)^{d / 2}\left(z^{2}+b\right)^{d / 2}} d y d z \\
\geq & c \int_{0}^{1} d y \frac{y}{\left(y^{2}+a\right)^{d / 2}} \int_{0}^{1} \frac{z^{2 H_{1}+1}}{\left(z^{2}+b\right)^{d / 2}} d z \\
= & c a^{-\frac{d}{2}+1} \int_{0}^{\frac{1}{\sqrt{a}}} \frac{u}{\left(u^{2}+1\right)^{d / 2}} d u b^{-\frac{d}{2}+1+H_{1}} \int_{0}^{\frac{1}{\sqrt{b}}} \frac{v^{2 H_{1}+1}}{\left(v^{2}+1\right)^{d / 2}} d v \\
\geq & c \frac{b^{H_{1}}}{a^{d / 2-1} b^{d / 2-1}} .
\end{aligned}
$$

This proves (4.20). In a similar way we can prove (4.19).

Let us consider (4.15) in the case when $d=k=2$, namely,

$$
\int_{-1}^{1} \cdots \int_{-1}^{1} \frac{\left|y_{1}\right|\left|z_{1}\right|\left|y_{1}-z_{1}\right|^{2 H_{1}}}{|y|^{d+2}} \frac{\left|y_{2}\right|\left|z_{2}\right|\left|y_{2}-z_{2}\right|^{2 H_{2}}}{|z|^{d+2}} d y d z
$$

where $0<H_{1}, H_{2}<1 / 2$. Applying (4.20) for $a=y_{2}^{2}, b=z_{2}^{2}$, we have

$$
\int_{-1}^{1} \cdots \int_{-1}^{1} \frac{\left|y_{1}\right|\left|z_{1}\right|\left|y_{1}-z_{1}\right|^{2 H_{1}}}{|y|^{d+2}} \frac{\left|y_{2}\right|\left|z_{2}\right|\left|y_{2}-z_{2}\right|^{2 H_{2}}}{|z|^{d+2}} d y d z \geq c \int_{-1}^{1} \int_{-1}^{1} \frac{z_{2}^{H_{1}}\left|y_{2}\right|\left|z_{2}\right|\left|y_{2}-z_{2}\right|^{2 H_{2}}}{y_{2}^{2} z_{2}^{2}} d y_{2} d z_{2}
$$

which is divergent. Thus we conjecture that $U(t, x)$ is in $L^{2}$ only if one or less Hurst exponent is less than $1 / 2$.

\section{The linear heat equation driven by fractional white noise}

In this section we consider the linear stochastic fractional heat equation

$$
\begin{gathered}
\frac{\partial U}{\partial t}(t, x)=\frac{1}{2} \Delta U(t, x)+W_{H}(t, x) ; \quad t \in(0, \infty), \quad x \in D \subset \mathbb{R}^{d} \\
U(0, x)=0 ; \quad x \in D \\
U(t, x)=0 ; \quad t \geq 0, \quad x \in \partial D
\end{gathered}
$$


Here $W_{H}(t, x)$ is the fractional white noise with Hurst parameter $H=\left(H_{0}, H_{1}, \ldots, H_{d}\right) \in$ $(0,1)^{d+1}, \Delta=\sum_{i=1}^{d} \frac{\partial^{2}}{\partial x_{i}^{2}}$ is the Laplace operator, $D \subset \mathbb{R}^{d}$ is a bounded open set with smooth boundary $\partial D$. We are looking for a solution $U:[0, \infty) \times \bar{D} \rightarrow(\mathcal{S})^{*}$ which is continuously differentiable in $(t, x)$ and twice continuously differentiable in $x$, i.e. belongs to $C^{1,2}((0, \infty) \times$ $\left.D ;(\mathcal{S})^{*}\right)$, and which satisfies $(5.1)$ in the strong sense (as an $(\mathcal{S})^{*}$-valued function).

Based on the corresponding solution in the deterministic case (with $W_{H}(t, x)$ replaced by a bounded deterministic function) it is natural to guess that the solution will be

$$
U(t, x)=\int_{0}^{t} \int_{D} W_{H}(s, y) G_{t-s}(x, y) d y d s
$$

where $G_{t-s}(x, y)$ is the Green function for the heat operator $\frac{\partial}{\partial t}-\frac{1}{2} \Delta$. It is well-known [D] that $G$ is smooth in $(0, \infty) \times D$ and that

$$
\left|G_{u}(x, y)\right| \sim u^{-d / 2} \exp \left(-\frac{|x-y|^{2}}{\delta u}\right) \quad \text { in }(0, \infty) \times D
$$

and

$$
\left|\frac{\partial G_{u}(x, y)}{\partial y_{i}}\right| \sim u^{-d / 2-1}\left|x_{i}-y_{i}\right| \exp \left(-\frac{|x-y|^{2}}{\delta u}\right) \quad \text { in }(0, \infty) \times D
$$

where the notation $X \sim Y$ means that

$$
\frac{1}{C} X \leq Y \leq C X \quad \text { in }(0, \infty) \times D
$$

for some positive constant $C<\infty$ depending only on $D$.

We use this to verify that $U(t, x) \in(\mathcal{S})^{*}$ for all $(t, x) \in[0, \infty) \times \bar{D}$ :

Using (3.27) we see that the expansion of $U(t, x)$ is

$$
\begin{aligned}
U(t, x) & \left.=\int_{0}^{t} \int_{D} G_{t-s}(x, y)\right) \sum_{k=1}^{\infty} M_{H} \eta_{k}(s, y) \mathcal{H}_{\varepsilon(k)}(\omega) d y d s \\
& =\sum_{k=1}^{\infty} b_{k}(t, x) \mathcal{H}_{\varepsilon(k)}(\omega),
\end{aligned}
$$

where

$$
b_{k}(t, x)=b_{\varepsilon^{(k)}}(t, x)=\int_{0}^{t} \int_{D} G_{t-s}(x, y) M_{H} \eta_{k}(s, y) d y d s
$$

In the following $C$ denotes a generic constant, not necessarily the same from place to place. From (3.28) we obtain that

$$
\begin{aligned}
\left|b_{k}(t, x)\right| & \leq C k^{\frac{2(d+1)}{3}} \int_{0}^{t} \int_{D} G_{t-s}(x, y) d y d s \\
& =C k^{\frac{2(d+1)}{3}} t
\end{aligned}
$$


Therefore

$$
\sum_{k=1}^{\infty} b_{k}^{2}(t, x)(2 \mathbb{N})^{-q \varepsilon^{(k)}} \leq C(t) \sum_{k=1}^{\infty} k^{\frac{4(d+1)}{3}}(2 k)^{-q}<\infty
$$

for $q>\frac{4(d+1)}{3}+1$.

Hence $U(t, x) \in(\mathcal{S})^{-q^{*}}$ for all $q>\frac{4(d+1)}{3}+1$, for all $t, x$.

In fact, this estimate also shows that $U(t, x)$ is uniformly continuous as a function from $[0, T] \times \bar{D}$ into $(\mathcal{S})^{*}$ for any $T<\infty$. Moreover, by the properties of $G_{t-s}(x, y)$ we get from (5.4) that

$$
\begin{aligned}
\frac{\partial U}{\partial t}(t, x)-\Delta U(t, x) & =\int_{0}^{t} \int_{D} W_{H}(s, y)\left(\frac{\partial}{\partial t}-\Delta\right) G_{t-s}(x, y) d y d s+W_{H}(t, x) \\
& =W_{H}(t, x)
\end{aligned}
$$

So $U(t, x)$ satisfies $(5.1)$.

Next we study the $L^{2}$-integrability of $U(t, x)$. In the standard white noise case $\left(H_{i}=\frac{1}{2}\right.$ for all $i$ ) the same solution formula (5.4) holds. In this case we see that the solution $U(t, x)$ belongs to $L^{2}(\mu)$ ( $\mu$ being the standard white noise measure) iff

$$
E_{\mu}\left[U^{2}(t, x)\right]=\int_{0}^{t} \int_{D} G_{t-s}^{2}(x, y) d y d s<\infty .
$$

Now, if $D \subset\left(-\frac{1}{2} R, \frac{1}{2} R\right)^{d}$ and we put $F=[-R, R]^{d}$,

$$
\begin{aligned}
\int_{0}^{t} \int_{D} G_{t-s}^{2}(x, y) d s d y & \sim \int_{0}^{t} \int_{D} s^{-d} \exp \left(-\frac{2 y^{2}}{\delta s}\right) d y d s \\
& \sim \int_{0}^{t}\left(\int_{F / \sqrt{s}} s^{-d / 2} \exp \left(-\frac{2 z^{2}}{\delta}\right) d z\right) d s .
\end{aligned}
$$

Hence, if $H_{i}=\frac{1}{2}$ for all $i=0,1, \ldots, d$ we have

$$
E_{\mu}\left[U^{2}(t, x)\right]<\infty \Longleftrightarrow d=1 .
$$

Now, consider the fractional case. Assume $\frac{1}{2}<H_{0}<1$ and because of (5.13) we may assume that at most one of the indices: $H_{1}, H_{2}, \ldots, H_{d}$ is less than $\frac{1}{2}$, say, $0<H_{1}<\frac{1}{2}$ (see also the remark at the end of Section 4). Then

$$
\begin{aligned}
& E\left[U^{2}(t, x)\right]=\int\left(M_{H} G_{t-.}(x, \cdot)(s, y)\right)^{2} d s d y \\
\leq & C \int_{0}^{t} \int_{0}^{t} \int_{D} \int_{D}\left|\frac{\partial G_{t-s}(x, y)}{\partial y_{1}}\right|\left|\frac{\partial G_{t-r}(x, z)}{\partial z_{1}}\right| \\
& \cdot|r-s|^{2 H_{0}-2}\left|y_{1}-z_{1}\right|^{2 H_{1}} \prod_{i=2}^{d}\left|y_{i}-z_{i}\right|^{2 H_{i}-2} d y_{1} \ldots d y_{d} d z_{1} \ldots d z_{d} d r d s . \\
& \sim \int_{0}^{t} \int_{0}^{t} \int_{D} \int_{D} r^{-d / 2-1} s^{-d / 2-1}\left|x_{1}-y_{1}\right|\left|x_{1}-z_{1}\right| \exp \left(-\frac{|x-y|^{2}}{\delta r}\right) \exp \left(-\frac{|x-z|^{2}}{\delta s}\right) \\
& \cdot|r-s|^{2 H_{0}-2}\left|y_{1}-z_{1}\right|^{2 H_{1}} \prod_{i=2}^{d}\left|y_{i}-z_{i}\right|^{2 H_{i}-2} d y_{1} \ldots d y_{d} d z_{1} \ldots d z_{d} d r d s .
\end{aligned}
$$


Note that

$$
\begin{array}{r}
\int_{-\frac{1}{2} R}^{\frac{1}{2} R} \int_{-\frac{1}{2} R}^{\frac{1}{2} R}\left|x_{1}-y_{1}\right|\left|x_{1}-z_{1}\right| \exp \left(-\frac{\left|x_{1}-y_{1}\right|^{2}}{\delta r}-\frac{\left|x_{1}-z_{1}\right|^{2}}{\delta s}\right) \\
\left|y_{1}-z_{1}\right|^{2 H_{1}} d y_{1} d z_{1} \leq \operatorname{Crs}\left(r^{H_{1}}+s^{H_{1}}\right)
\end{array}
$$

Using (see [MMV], Inequality (2.1))

$$
\int_{R} \int_{R}|f(x)||g(y)||x-y|^{2 H-2} d x d y \leq C|| f\left\|_{L^{1 / H}}\right\| g \|_{L^{1 / H}}
$$

we have

$$
\begin{aligned}
& \prod_{i=2}^{d} \int_{-\frac{1}{2} R}^{\frac{1}{2} R} \int_{-\frac{1}{2} R}^{\frac{1}{2} R} \exp \left(-\frac{\left|x_{i}-y_{i}\right|^{2}}{\delta r}-\frac{\left|x_{i}-z_{i}\right|^{2}}{\delta s}\right)\left|y_{i}-z_{i}\right|^{2 H_{i}-2} d y_{i} d z_{i} \\
& \leq C \prod_{i=2}^{d}\left\{\left[\int_{-\frac{1}{2} R}^{\frac{1}{2} R} \exp \left(-\frac{\left|x_{i}-y_{i}\right|^{2}}{H_{i} \delta r}\right) d y_{i}\right]^{H_{i}}\left[\int_{-\frac{1}{2} R}^{\frac{1}{2} R} \exp \left(-\frac{p_{i}\left|x_{i}-z_{i}\right|^{2}}{H_{i} \delta s}\right) d z_{i}\right]^{H_{i}}\right\} \\
& \sim(r s)^{\frac{1}{2} \sum_{i=2}^{d} H_{i}} .
\end{aligned}
$$

Substituting (5.17) into (5.14) we have

$$
\begin{gathered}
E\left[U^{2}(t, x)\right] \leq C \int_{0}^{t} \int_{0}^{t}(r s)^{-\frac{d}{2}+\frac{1}{2} \sum_{i=2}^{d} H_{i}}\left(r^{H_{1}}+s^{H_{1}}\right)|r-s|^{2 H_{0}-2} d r d s<\infty \\
\quad \text { if } \quad d / 2-\frac{1}{2} \sum_{i=2}^{d} H_{i}<1 \\
2-2 H_{0}+2\left(\frac{d}{2}-\sum_{i=2}^{d} \frac{1}{2} H_{i}\right)-H_{1}<2
\end{gathered}
$$

We obtain from this that

$$
E\left[U^{2}(t, x)\right]<\infty \quad \text { if }\left[\left(2 H_{0}+H_{1}\right) \wedge 2\right]+\sum_{i=2}^{d} H_{i}>d .
$$

Now let $1 / 2<H_{i}<1$ for all $1 \leq i \leq d$. Then

$$
\begin{aligned}
& E\left[U^{2}(t, x)\right]=\int\left(M_{H} G_{t-.}(x, \cdot)(s, y)\right)^{2} d s d y \\
& \sim \int_{0}^{t} \int_{0}^{t} \int_{D} \int_{D}\left|G_{t-s}(x, y) G_{t-r}(x, z)\right| \\
& \cdot|r-s|^{2 H_{0}-2} \prod_{i=1}^{d}\left|y_{i}-z_{i}\right|^{2 H_{i}-2} d y_{1} \ldots d y_{d} d z_{1} \ldots d z_{d} d r d s . \\
& \sim \int_{0}^{t} \int_{0}^{t} \int_{D} \int_{D} r^{-d / 2} s^{-d / 2} \exp \left(-\frac{|x-y|^{2}}{\delta r}\right) \exp \left(-\frac{|x-z|^{2}}{\delta s}\right) \\
& \cdot|r-s|^{2 H_{0}-2} \prod_{i=1}^{d}\left|y_{i}-z_{i}\right|^{2 H_{i}-2} d y_{1} \ldots d y_{d} d z_{1} \ldots d z_{d} d r d s .
\end{aligned}
$$


By (5.16), we have

$$
\begin{aligned}
& \prod_{i=1}^{d} \int_{-\frac{1}{2} R}^{\frac{1}{2} R} \int_{-\frac{1}{2} R}^{\frac{1}{2} R} \exp \left(-\frac{\left|x_{i}-y_{i}\right|^{2}}{\delta r}-\frac{\left|x_{i}-z_{i}\right|^{2}}{\delta s}\right)\left|y_{i}-z_{i}\right|^{2 H_{i}-2} d y_{i} d z_{i} \\
& \leq C \prod_{i=1}^{d}\left\{\left[\int_{-\frac{1}{2} R}^{\frac{1}{2} R} \exp \left(-\frac{\left|x_{i}-y_{i}\right|^{2}}{H_{i} \delta r}\right) d y_{i}\right]^{H_{i}}\left[\int_{-\frac{1}{2} R}^{\frac{1}{2} R} \exp \left(-\frac{p_{i}\left|x_{i}-z_{i}\right|^{2}}{H_{i} \delta s}\right) d z_{i}\right]^{H_{i}}\right\} \\
& \sim(r s)^{\frac{1}{2} \sum_{i=1}^{d} H_{i}} .
\end{aligned}
$$

Substituting (5.22) into (5.21) we have

$$
\begin{gathered}
E\left[U^{2}(t, x)\right] \leq C \int_{0}^{t} \int_{0}^{t}(r s)^{-\frac{d}{2}+\frac{1}{2} \sum_{i=1}^{d} H_{i}}|r-s|^{2 H_{0}-2} d r d s<\infty \\
\quad \text { if } 2 H_{0}+\sum_{i=1}^{d} H_{i}>d .
\end{gathered}
$$

We summarize what we have proved:

Theorem 5.1 a) For any space dimension $d$ there is a unique strong solution $U(t, x)$ : $[0, \infty) \times D \rightarrow(\mathcal{S})^{*}$ of the fractional heat equation (5.1)-(5.3). The solution is given by

$$
U(t, x)=\int_{0}^{t} \int_{D} W_{H}(s, y) G_{t-s}(x, y) d y d s .
$$

It belongs to $C^{1,2}\left((0, \infty) \times D \rightarrow(S)^{*}\right) \cap C\left([0, \infty) \times \bar{D} \rightarrow(S)^{*}\right)$.

b) If $0<H_{1}<\frac{1}{2}, \frac{1}{2}<H_{i}<1$ for $i=0,2,3, \ldots$, $d$ and

$$
\left[\left(2 H_{0}+H_{1}\right) \wedge 2\right]+\sum_{i=2}^{d} H_{i}>d
$$

then $U(t, x) \in L^{2}(\mu)$ for all $t \geq 0, x \in \bar{D}$.

c) If $\frac{1}{2}<H_{i}<1$ for $i=0,1, \ldots, d$ and

$$
2 H_{0}+\sum_{i=1}^{d} H_{i}>d
$$

then $U(t, x) \in L^{2}(\mu)$ for all $t \geq 0, x \in \bar{D}$.

Acknowledgement: We are grateful to the referee for the careful reading and insight comments which lead to many improvements of the earlier version. Hu is supported in part by the National Science Foundation under Grant No. DMS 0204613 and No. EPS-9874732, matching support from the State of Kansas and General Research Fund of the University of Kansas. Zhang is supported by the British EPSRC (grant no. GR/R91144/01). 


\section{References}

[AF] A. Aharong and J. Feder (editors): Fractals in Physics. Essays in honor of Benoit B. Mandelbrot. North-Holland 1990.

[D] E.B.Davis: Heat Kernels and Spectral Theory. Cambridge University Press, 1989.

[DHP] T.E. Duncan, Y. Hu and B. Pasik-Duncan: Stochastic calculus for fractional Brownian motion. SIAM J. Control \& Optim 38 (2000), 582-612.

[EvdH] R.J. Elliott and J. van der Hoek: A general fractional white noise theory and applications to finance. Manuscript 2000.

[H1] Y. Hu: Heat equation with fractional white noise potentials. Applied Mathematics and Optimization, 43 (2001), 221-243.

[H2] Y. Hu: A class of stochastic partial differential equations driven by fractional white noise. In F. Gesztesy et al. (editors): Stochastic Processes, Physics and Geometry: New Interplays II. Conference Proceedings, Canadian Mathematical Society, Vol. 29, AMS 2000, pp. 317-325.

[H3] Y. Hu, Probability structure preserving and absolute continuity. Ann. Inst. H. Poincaré Probab. Statist. 38 (2002), no. 4, 557-580.

[HKPS] T. Hida, H.-H. Kuo, J. Potthoff and L. Streit: White Noise Analysis. Kluwer 1993.

$[\mathrm{H} \varnothing]$ Y. Hu and B. Øksendal: Fractional white noise calculus and applications to finance. Preprint, University of Oslo 10/1999.

[HØUZ] H. Holden, B. Øksendal, J. Ubøe and T. Zhang: Stochastic Partial Differential Equations. Birkhäuser 1996.

[HØZ1] Y. Hu, B. Øksendal and T. Zhang: Stochastic partial differential equations driven by multiparameter fractional white noise. In F. Gesztesy et al. (editors): Stochastic Processes, Physics and Geometry: New Interplays II. Conference Proceedings, Canadian Mathematical Society, Vol. 29, AMS 2000, pp. 327-337.

[HØZ2] Y. Hu, B. Øksendal and T. Zhang: Stochastic fractional potential theory. Papers on analysis, 169-180, Rep. Univ. Jyväskylä Dep. Math. Stat., 83, Univ. Jyväskylä, 2001.theory. Manuscript 2001.

[Ko] A.N. Kolmogorov: Selected Works, Vol. II: Probability Theory and Mathematical Statistics (editor A.N. Shiryaev). Kluwer 1992.

[Ku] H.-H. Kuo: White Noise Distribution Theory. CRC Press 1996.

[M] B.B. Mandelbrot: The Fractal Geometry of Nature. Freeman 1983.

[MvN] B.B. Mandelbrot and J.W. van Ness: Fractional Brownian motions, fractional noises and applications. SIAM Rev. (1968), 422-437. 
[MMV] J. Mëmin, Yu. Mishura and E. Valkeila, Inequalities for the moments of Wiener integrals with respect to a fractional Brownian motion. Statist. Probab. Lett. 51 (2001), 197-206.

[ØZ] B. Øksendal and T. Zhang: Multiparameter fractional Brownian motion and quasilinear stochastic partial differential equations. Stochastics and Stochastics Reports 71 (2001), 141-163.

[PT] V. Pipiras and M. S. Taqqu: Integration questions related to fractional Brownian motion. Probability Theory and Related Fields 118 (2000), 251-291.

[SKM] S. G. Samko, A. A. Kilbas and O. I. Marichev, Fractional Integrals and Derivatives, Theory and Applications. Gordon and Breach Science Publishers, 1993.

[S] A. Shiryaev: Essentials of Stochastic Finance. World Scientific 1999. 